\title{
An interference-resilient IIoT solution for measuring the effectiveness of industrial processes
}

\author{
Angel C. Herrero*, Francisco J. Martinez*, Piedad Garrido*, \\ Julio A. Sanguesa ${ }^{\dagger}$, Carlos T. Calafate \\ *University of Zaragoza, Spain - acherrero, f.martinez, piedad $\} @$ unizar.es \\ ${ }^{\dagger}$ Centro Universitario de la Defensa, Spain — jsanguesa@unizar.es \\ ‡DISCA - Universitat Politècnica de València, Spain — calafate@disca.upv.es
}

\begin{abstract}
The development and deployment of the so-called Industrial Internet of Things (IIoT) have significantly increased the control and monitoring capabilities of companies, and thus their potential productivity. In this paper, we propose the use of Raspberry $\mathrm{Pi}$ devices in industrial environments to measure productivity parameters. Our proposal can economically and efficiently gather data related with the availability and productivity of industrial machinery. However, since low-cost devices are prone to suffer the negative effects of electromagnetic interferences, we additionally propose an alternative to prevent signal alterations caused by them. More specifically, we propose a filtering mechanism called Smart Coded Filter (SCF), which eliminates wrong signals caused by electromagnetic interferences, and, therefore, highly improves the accuracy when estimating the availability metric. Results obtained demonstrate that our lowcost device provided with the SCF completely ignores $100 \%$ of wrong availability data, while reducing up to $70 \%$ the number of records stored into the database.
\end{abstract}

Index Terms-Industrial Internet of Things, OEE, Low-Cost devices, Raspberry Pi, Electromagnetic Interferences.

\section{INTRODUCTION}

The Industry 4.0 concept was initially coined by the German government within its High-Tech Strategy [1]. In particular, it focuses on enterprise-wide business process automation to create smart factories, thus fostering the development of new business models to enhance the value chain thanks to the application of Big Data and Machine Learning strategies to the industrial environment. Industry 4.0, basically, seeks to incorporate the information and communication technologies to allow the customization of products and flexible mass production.

One important concept related to Industry 4.0 is the socalled Industrial Internet of Things (IIoT), which refers to the use of automated and connected machines, devices, and sensors in industrial environments. IIoT not only enables remote condition monitoring, but also increases the efficiency and reliability of enterprises and industries.

In that industrial environment, the Overall Equipment Effectiveness (OEE) is an useful metric to accurately estimate the productivity of industries. This metric can be measured in real time by means of the IoT paradigm, where smart devices can be deployed to collect important data, thus providing industry owners with useful information to make the right decisions to improve productivity while reducing costs.
OEE considers three key factors which affect the equipment effectiveness. They are: (i) availability, (ii) performance, and (iii) quality. Fortunately, there currently exist IoT-based sensors and devices which allow us to gather the data required to accurately measure all these factors, and thus estimate the OEE. However, low-cost low-power IoT devices can present problems due to electromagnetic interferences (EMIs) commonly present in hostile electromagnetic environments such as industries, where a plethora of electronic components and power supply units can cause EMIs.

In this paper, we propose a system, based on Raspberry Pi, which is specially designed to monitor the productivity and effectiveness of industries by means of the real-time estimation of the OEE. In addition, we propose the Smart Coded Filter (SCF), designed to prevent the problems that EMIs cause in this type of low-cost devices.

So far, several works related to OEE estimation can be found in the literature. Kao et al. [2] proposed a novel metric coined as Predictive Overall Equipment Effectiveness (POEE). In particular, they seek to monitor and assess the forthcoming effectiveness of a single tool. The POEE is composed of two parts: (i) the deterministic effectiveness, such as the preventive maintenance, engineering experiments, as well as job scheduling, and (ii) the predictive effectiveness in terms of the extra production time due to the abnormal tool condition or undesired product quality. Krachangchan and Thawesaengskulthai [3] thoroughly studied the problems related to breakdowns, equipment defects, and poor working conditions in a tobacco company. The purpose of their work was to reduce time losses, and so improve performance through the implementation of Total Productive Maintenance (TPM) and Reliability Centered Maintenance (RCM) by using Failure Modes and Effects Analysis (FMEA). After the implementation of their approach, the performance rate increased from $75 \%$ to $79 \%$, which also enhanced OEE from $66 \%$ to $72 \%$. Unlike our work, none of these proposals considered the use of low-lost devices, studied the effect of EMIs, or the possibility of remotely obtaining real-time OEE.

Regarding the use of low-cost low-power devices in industrial environments, Weiss [4] presented a low-cost radar for near-range, which employs nanosecond pulses in the $5.8 \mathrm{GHz}$ industrial, scientific, and medical (ISM)-band. Experimental 


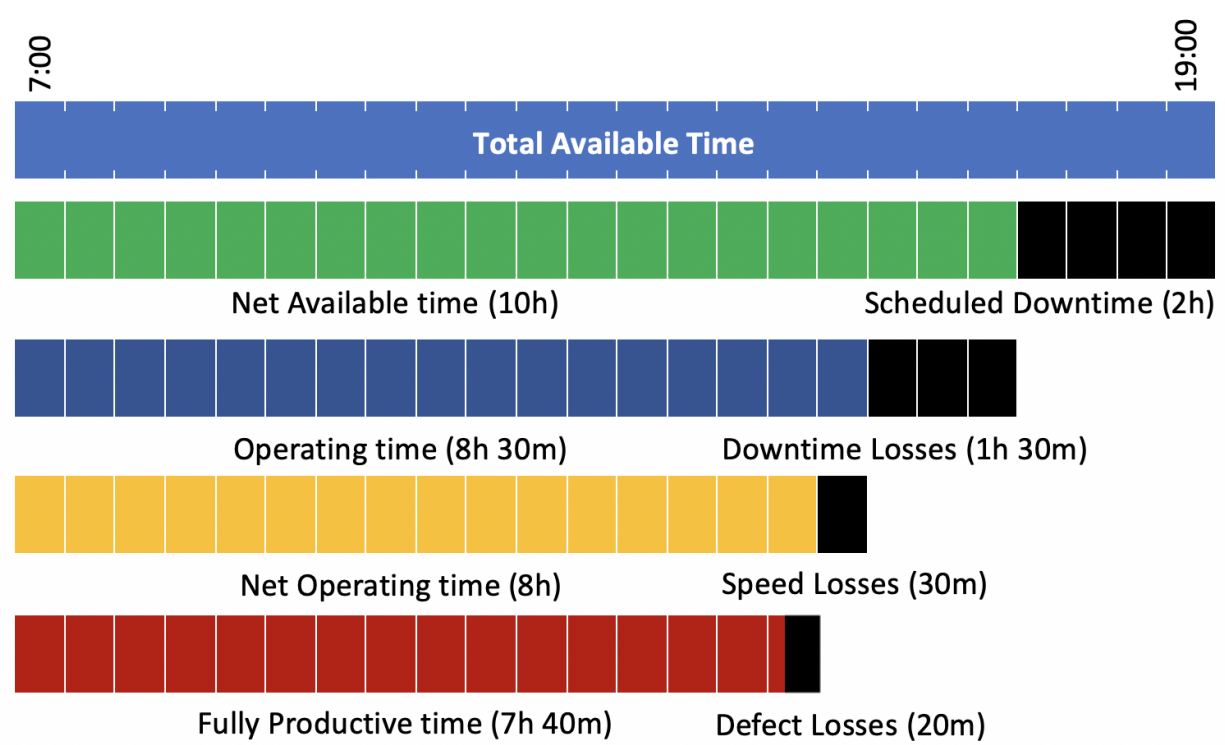

Fig. 1: Example of OEE and its parameters during a full working day

results showed good performance in industrial environments, i.e., it showed an accuracy about $10 \mathrm{~mm}$ over a distance of $10 \mathrm{~m}$. Haus et al. [5] studied several cost-effective piezoresistive materials to implement low-cost tactile sensor matrices for physical human robot interaction (pHRI) applications in industrial manufacturing environments. In particular, they modeled the input-output behavior by using a machine learning approach to determine the best material and a cheaper alternative.

Unlike previously presented works, our proposal also mitigates the effect of electromagnetic interferences in low-cost low-power devices, which are prone to occur in industrial environments, and especially affect this kind of devices. Therefore, preventing them represents a challenge. Our proposal presents a low-cost system which provides sensing and communication capabilities to accurately estimate OEE. In particular, our approach can monitor the availability of machinery, while ignoring erroneous signals due to the electronic noise caused by EMIs.

\section{BACKGROUND: OVERALL EQUIPMENT EFFECTIVENESS}

The main objective of our proposal is measuring one of the parameters that contribute to calculating real-time OEE using low-cost devices. OEE reflects how the equipment of a industry is performing to meet the production demanding expectations [6]. This indicator enables us to better understand the main reasoning behind deficient performance and, therefore, it provides the basis for establishing improvement priorities to fix problems.

Figure 1 shows the factors used to calculate OEE. They are:

- Availability metric, which measures the percentage of time that the machine is really available to work.

- Performance metric, which represents the percentage of time that the machine is available to work, but without considering the time lost due to speed losses.
- Quality metric, which measures the percentage of time that the machine is fully productive, subtracting the time lost due to defect losses (i.e., the time taken to produce defective products).

Equation 1 details how the OEE is calculated.

$$
O E E=\text { Availability } \cdot \text { Performance } \cdot \text { Quality }
$$

where Availability $=\frac{O T}{N A T}$, Performance $=\frac{N O T}{O T}$, and Quality $=\frac{F P T}{N O T}$

The OEE, besides allowing the evaluation of equipment effectiveness, can also be used to compare different industry production units. In particular, OEE provides significant information to optimize the use of resources, as well as the adoption of better practices in the industry [7].

Concerning the OEE calculation, for the sake of clarity, Figure 1 depicts an example of production data during one day. In particular, it shows the total scheduled time, and the time lost due to different causes (i.e., scheduled shutdowns, inactivity, speed losses, and defective products). These values allow us to calculate the parameters affecting availability, performance, and quality, and ultimately, the OEE value. Finally, equation 2 shows how the OEE is calculated considering the data presented in Figure 1.

$$
O E E=\frac{8.5}{10} \cdot \frac{8}{8.5} \cdot \frac{7.66}{8}=0.766
$$

The values return an OEE of $76.6 \%$, thus showing that, although the overall equipment effectiveness is very acceptable, improvements should mainly focus on those issues related to increasing the availability, i.e., reducing time losses due to unscheduled inactivity. 


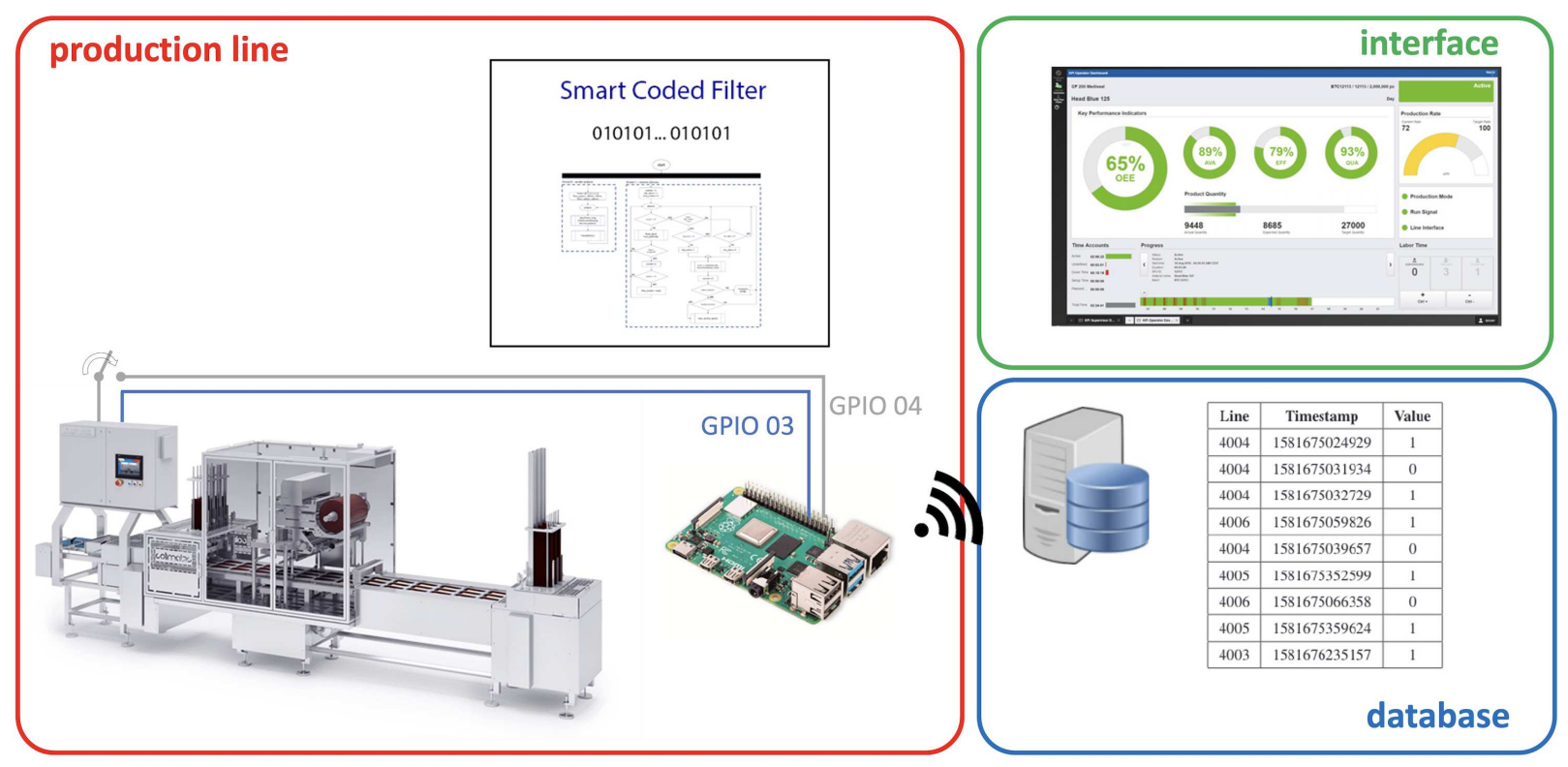

Fig. 2: System configuration.

\section{LOW-COST MeAsurement of THE MAChinery AVAILABILITY}

In this paper we focus on solving the problems related to the measurement of the OEE availability factor in real time. In particular, our approach was validated in two different industrial environments: (i) a meat product processing industry, that performs several operations with a variety of products, mainly ham, and (ii) a cheese factory that produces cheese in various formats. In this section, we present the system configuration, detailing how it operates.

As stated before, our system measures one of the factors involved in the OEE calculation, in particular, the availability parameter, which is obtained by processing the availability signals obtained from any machine. Our proposal improves many current systems, since this parameter has to be manually introduced by operators, in many cases.

Figure 2 shows the system configuration. More specifically, it presents the different elements involved in gathering the data related to availability. They are: (i) industrial equipment. In the example, we show a cheese vacuum sealer, although our system can monitor the availability of any machine, (ii) a Raspberry $\mathrm{Pi}$, which receives signals from the machine, processes them, and sends data to the database via a wireless connection, (iii) the database, which stores the signals collected, and (iv) a dashboard, which enables the visualization of the overall effectiveness.

After operating the proposed system in a real industrial environment, that is, a cheese packaging plant, we found EMIs causing noise in the signals, since the system records much more availability signals than those that actually occur.

Particularly, with the purpose of quantifying the erroneous signals the system records, we carefully checked several production shifts, although, in this paper, we only present six
TABLE I: Results obtained in different working days

\begin{tabular}{|c|c|c|c|}
\hline Day & \#Real signals & \#Stored signals & error \\
\hline $\mathbf{1}$ & 82 & 282 & $244 \%$ \\
\hline $\mathbf{2}$ & 116 & 172 & $48 \%$ \\
\hline $\mathbf{3}$ & 28 & 84 & $200 \%$ \\
\hline $\mathbf{4}$ & 50 & 72 & $44 \%$ \\
\hline $\mathbf{5}$ & 28 & 50 & $79 \%$ \\
\hline
\end{tabular}

different working days. During these observations we analyzed the availability of the sealing machine, according to the data provided by machine operators, and compared them against the data obtained and stored by our system.

Table I quantifies the effect of electromagnetic interferences in the availability signal by comparing the signals recorded by the device for these days with the real availability data. In particular, the table shows the number of real availability signals, which includes both the number of shutdowns and unscheduled stops, as well as the number of signals already detected. As shown, the error committed greatly varies (ranging from 44 to $244 \%$ ), making it very difficult to predict. Moreover, it is clearly unacceptable, thus making it absolutely necessary to address the problems due to EMIs.

\section{Proposed Approach to Increase Resilience to ELECTROMAGNETIC INTERFERENCE (SCF)}

As stated above, we corroborated that our system can monitor the machines' availability, as required to estimate the OEE, but we also detected that the number of signals recorded was significantly higher than expected due to electromagnetic interferences (EMIs). On this basis, in this work we additionally designed the Smart Coded Filter (SCF), which avoids wrong availability signals, and thus improves the precision of our system. 


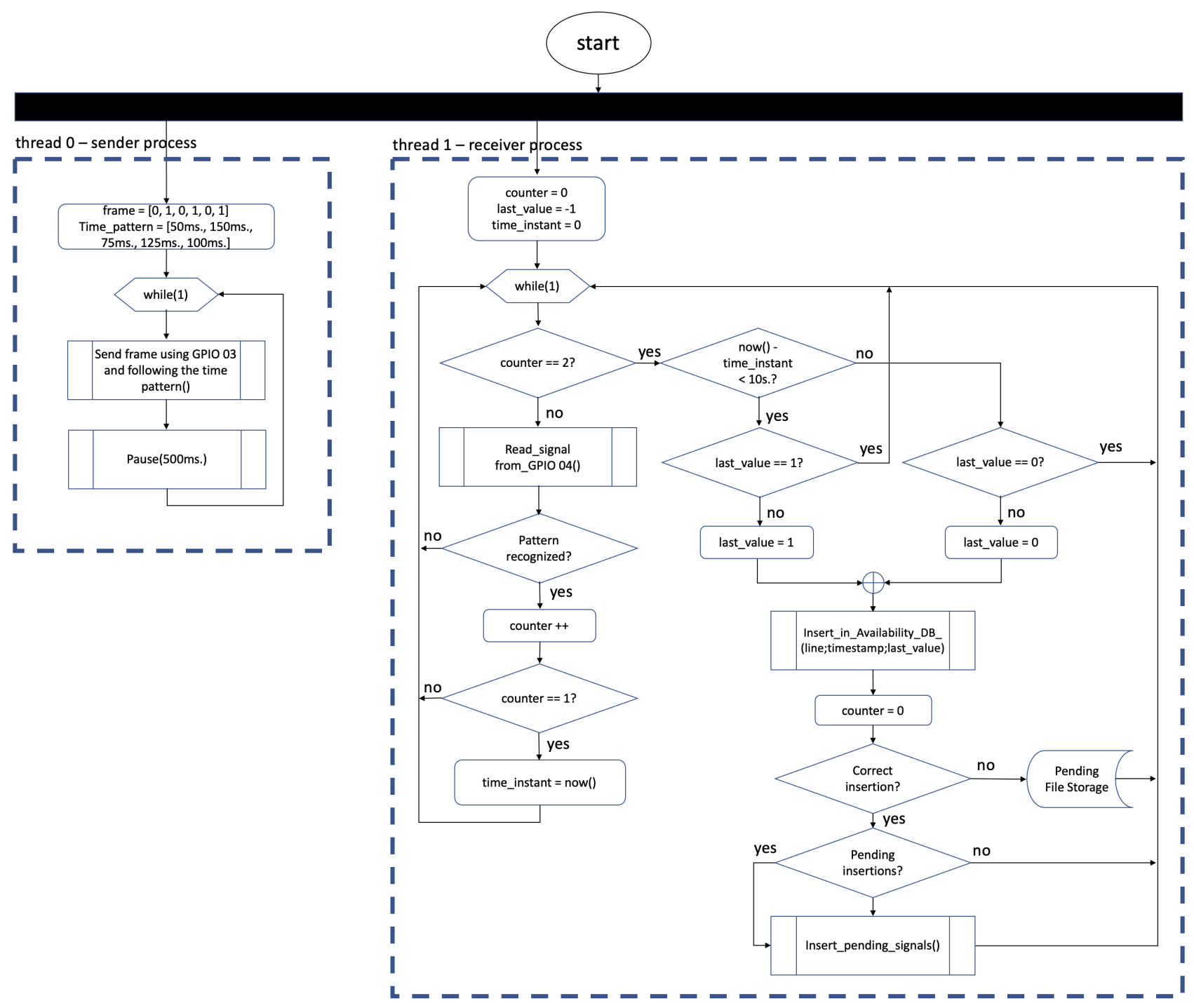

Fig. 3: Flow chart of the Smart Coded Filter.

Figure 3 presents a flow chart which summarizes how SCF operates. In particular, it shows the two different processes that run in parallel on the Raspberry Pi. On the one hand, the sender process continually sends (every second), using the GPIO 03 port, the frame $(0-1-0-1-0-1)$ by following a predetermined duration per bit $(50 \mathrm{~ms}, 150 \mathrm{~ms}, 75 \mathrm{~ms}, 125 \mathrm{~ms}$, and $100 \mathrm{~ms})$, i.e., the system sends a ' 0 ' during $50 \mathrm{~ms}$., then sends a ' 1 ' during $150 \mathrm{~ms}$., a '0' during $75 \mathrm{~ms}$., etc., and waits for $500 \mathrm{~ms}$ to repeat the process again. On the other hand, the receiver process continually monitors the GPIO 04 port waiting to recognize the frame sent by the sender process, since the frame could be only received correctly when the sealer switches the circuit on, in other words, when the machine is available.

In order to greatly increase the reliability of the system, it only considers that an availability signal is correct when the frame is correctly received twice in the last 10 seconds (note the counter parameter in the flow chart). Otherwise, it means that the machine is currently unavailable. This double check ensures that the signals received are not fictitiously generated by electromagnetic noise.

Once the signal has been identified, it is stored in the availability database. If data is not inserted correctly, usually due to a lack of connectivity, the signal goes to the Pending File Storage, and the process will go back to waiting for a new signal. Conversely, i.e., in case of available connection, the system will check whether there are signals stored in the Pending File Storage, and will insert them into the database.

The data stored in the availability database basically contains the following information: (i) line, a four-digit code that identifies the machine from which data are obtained, (ii) timestamp, which determines the time instant when the signal is received, and (iii) signal value, which is 1 or 0 when the machine is available or not, respectively. It is also worth mentioning that the last_value parameter prevents the system from continuously inserting duplicate values into the database, since once an availability (or unavailability) signal has been 
TABLE II: Available and unavailable times for the different working days

\begin{tabular}{|c|c|c|c|c|c|c|c|}
\hline \multirow{2}{*}{ Day } & \multicolumn{3}{|c|}{ System w/ SCF } & \multicolumn{4}{|c|}{ System w/o SCF } \\
\hline & Available time & Unavailable time & Availability ratio & Available time & Unavailable time & Availability ratio & Error \\
\hline 1 & 11h 33'29”' & $3 \mathrm{~h} 26^{\prime} 31^{\prime \prime}$ & 0.771 & 10h 23'48”' & 4h $36^{\prime} 12^{\prime \prime}$ & 0.693 & $10.05 \%$ \\
\hline 2 & 11h 50'46" & 3h 09' 14" & 0.790 & $10 \mathrm{~h} 02^{\prime} 28^{\prime \prime}$ & 4h $57^{\prime} 32^{\prime \prime}$ & 0.669 & $15.24 \%$ \\
\hline 3 & $12 \mathrm{~h} 26^{\prime} 35^{\prime \prime}$ & $2 \mathrm{~h} 33^{\prime} 25^{\prime \prime}$ & 0.830 & 11h 39'50"' & $3 \mathrm{~h} 20^{\prime} 10^{\prime \prime}$ & 0.778 & $6.26 \%$ \\
\hline 4 & 12h 40'05' & $2 \mathrm{~h} \mathrm{19'55^{ \prime \prime }}$ & 0.845 & $8 \mathrm{~h} \mathrm{46} 26^{\prime \prime}$ & $6 h 13$ '34" & 0.585 & $30.74 \%$ \\
\hline 5 & 12h 04'08" & 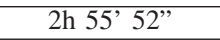 & 0.805 & 11h 16' 42" & $3 \mathrm{~h} \mathrm{43}$ '18' & 0.752 & $6.55 \%$ \\
\hline
\end{tabular}

stored, the next signal to store should not be the same.

The purpose of SCF is to discard received signals that do not represent the machine's availability. According to this, erroneous signals are filtered immediately by the system provided they do not comply with the SCF protocol. Consequently, SCF prevents fictitious availability or unavailability values from being inserted into the database. The effectiveness of SCF was assessed by comparing the data provided by operators to the availability signals recorded by our system. This comparison demonstrated the accuracy and reliability of our approach, since SCF ignored $100 \%$ of wrong signals.

Table II presents the available and unavailable times, as well as the availability ratios and the error values, comparing our system with and without our SCF approach. As shown, availability times and ratios are quite different for both approaches. In fact, the error introduced by the system without using SCF varies from $6.26 \%$ to $30.74 \%$. Hence, this issue additionally demonstrates the need for avoiding wrong signals due to EMIs.

Figures 4 and 5 present a visual comparison of the availability signals detected by both approaches, i.e., the one including the SCF, and the one without it, respectively. Due to space limitations, we only include data from days 3 and 4, because they can be considered quite interesting, as they presented the lowest and highest errors in terms of availability ratio, although it is worth mentioning that the results for the rest of days are similar. Note that the periods in which the machine is available are colored in light blue (w/ SCF) and light red (w/o SCF), whereas the inactivity periods appear in white.

Regarding the system including SCF, which accurately reflects the availability of the machine, we can easily observe the product changeovers (i.e., when the production changes between whole cheeses, wedges, quarters, or slices). In particular we can find them at 11:10, 19:30 and 19:50, in Figure 4a, and 8:15, 8:25, 11:20, 14:10, 20:00, and 21:10 in Figure 5a. In addition, scheduled downtimes can be identified starting from 13:50 (in Day 3), as well as at 14:50 and 18:10 (in Day 4). Finally, note that there are two unavailability slots during Day 3 due to minor breakdowns. More specifically, they can be found at the beginning of the day (from 7:00 to 7:45), and also from 11:30 to 13:00. However, electromagnetic noise provoked that the system without SCF wrongly considered that, during the first shutdown slot, the machine was mainly available (see Figure 4b).

As for the effect of EMIs, and according to data previously presented on Table II, the two approaches behave differently. In particular, they present similar results in terms of availability in Day 3, whereas noticeable differences appear in Day 4.
The effects of EMIs are not only reflected in sudden changes of availability, but also in wrong availabilities for longer periods, which clearly do not express the real availability of the machine. Figure $4 \mathrm{~b}$ shows erroneous signals at 7:15, $8: 45,11: 35,12: 10,17: 15$, and 19:00 (see Figure 4b). These alterations can also be observed in Day 4 (see Figure 5b), as there are wrong signals starting from 7:15, 7:30, 13:00, 17:05, and 19:20. Although the availability errors should be fixed to correctly estimate OEE, there is an additional benefit of using $\mathrm{SCF}$, since the number of signals stored in the database is considerably reduced using our proposal (up to $70 \%$ fewer accesses). This is also an important issue, since having a high amount of transactions in the database is clearly inefficient and can overload the system. In summary, the results obtained demonstrated that, using the availability monitoring system, and including the Smart Coded Filter we have proposed, we are capable of correctly measuring the availability ratio, required to estimate $\mathrm{OEE}$, since our approach completely ignores wrong signals due to the electromagnetic noise.

\section{Conclusions}

Estimating OEE in real time, by using IIoT, enables business managers to detect which aspects they should study in detail to improve the efficiency of the production process, and thus increase their benefits. In this paper, we proposed a system based on low-cost devices for accurately estimate the availability of any industrial machine.

Additionally, we also addressed the problems caused by EMIs, which have a special impact on this type of low-power devices. More specifically, we propose a filtering mechanism, namely the Smart Coded Filter, which completely ignores erroneous signals caused by EMIs, thus enabling an accurate OEE estimation at a considerably lower cost than other proprietary systems with similar characteristics.

Results demonstrated that SCF ignores $100 \%$ of wrong availability data, hence avoiding all the signal alterations due to electromagnetic noise. In addition, our system reduces up to $70 \%$ the number of accesses, and so the number of records stored into the database.

\section{ACKNOWLEDGMENTS}

This work has been partially supported by the Government of Aragón and the European Social Fund "Construyendo Europa desde Aragón” (T40_20D Research Group), as well as the "Ministerio de Ciencia, Innovación y Universidades, Programa Estatal de Investigación, Desarrollo e Innovación Orientada a los Retos de la Sociedad, Proyectos I+D+i 2018", Spain, under Grant RTI2018-096384-B-I00. 


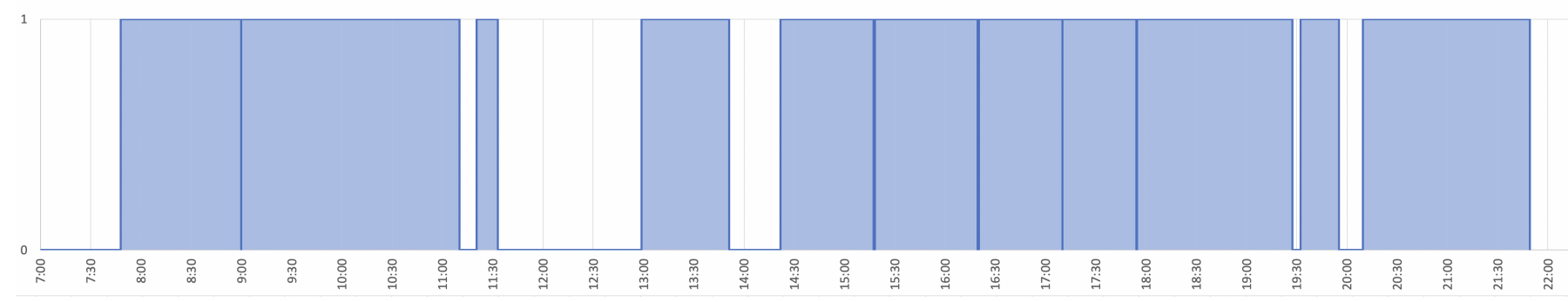

(a)

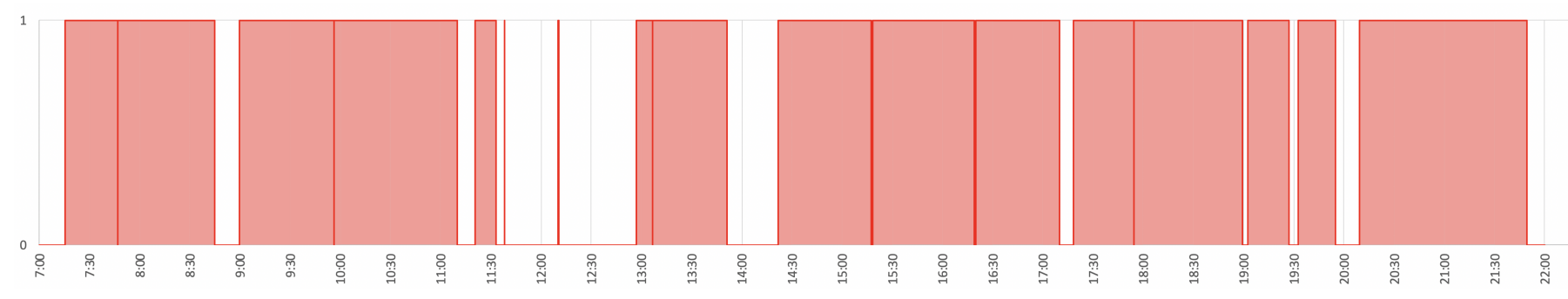

(b)

Fig. 4: Availability signals obtained during Day 3: (a) using the proposed system with SCF and (b) without including the SCF approach.

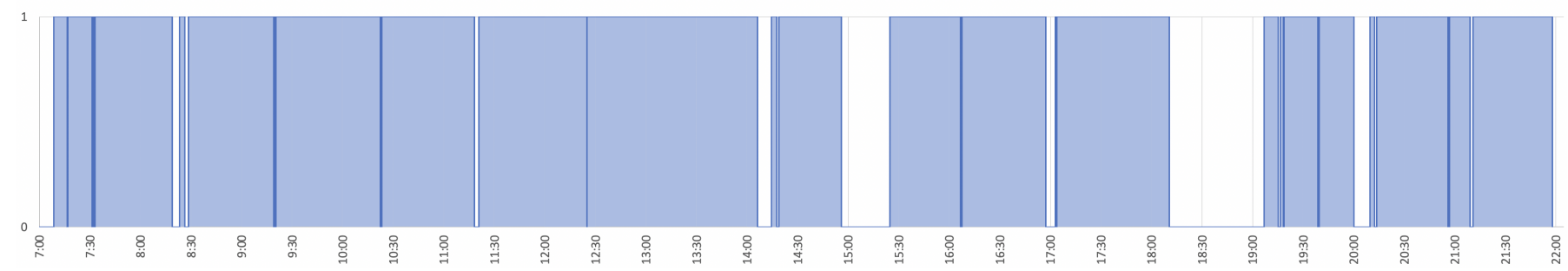

(a)

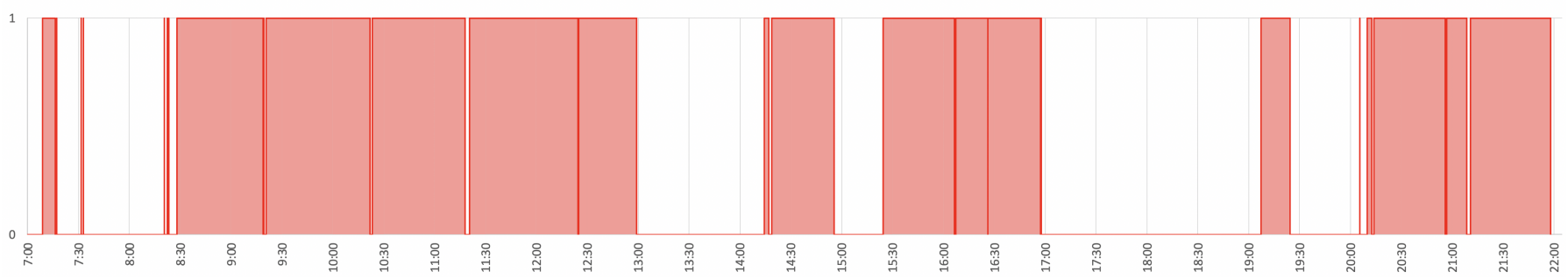

(b)

Fig. 5: Availability signals obtained during Day 4: $(a)$ using the proposed system with SCF and $(b)$ without including the SCF approach.

\section{REFERENCES}

[1] Federal Ministry of Education and Research, "High-Tech Strategy," 2017. [Online]. Available: https://ec.europa.eu/growth/tools-databases/ dem/monitor/sites/default/files/DTM_Industrie\%204.0.pdf

[2] Y. Kao, S. Chang, S. Dauzere-Peres, and J. Blue, "Opportunity for improving fab effectiveness by predictive overall equipment effectiveness (poee)," in e-Manufacturing and Design Collaboration Symposium (eMDC), Sep. 2016, pp. 1-4.

[3] K. Krachangchan and N. Thawesaengskulthai, "Loss time reduction for improve overall equipment effectiveness (oee)," in 5th International Conference on Industrial Engineering and Applications (ICIEA), April 2018, pp. 396-400.
[4] M. Weiss, "Low-cost, low-power nanosecond pulse radar for industrial applications with mm accuracy," in International Symposium on Electron Devices for Microwave and Optoelectronic Applications (EDMO) (Cat. No.01TH8567), Nov 2001, pp. 199-204.

[5] J. N. Haus, A. Muxfeldt, and D. Kubus, "Material comparison and design of low cost modular tactile surface sensors for industrial manipulators," in IEEE 21st International Conference on Emerging Technologies and Factory Automation (ETFA), Sep. 2016, pp. 1-7.

[6] R. M. Williamson, "Using overall equipment effectiveness: the metric and the measures," Strategic Work System, Inc, pp. 1-6, 2006.

[7] M. Fekri Sari and S. Avakh Darestani, "Fuzzy overall equipment effectiveness and line performance measurement using Artificial Neural Network," Journal of Quality in Maintenance Engineering, vol. 25, no. 2, pp. 340354, 2019. 